Теорія Ймовір. та Матем. Статист. Вип. 76, 2007
Theor. Probability and Math. Statist.

No. 76, 2008, Pages 1-13

S 0094-9000(08)00726-6

Article electronically published on July 10, 2008

\title{
AN ESTIMATE FOR THE RUIN PROBABILITY IN A MODEL WITH VARIABLE PREMIUMS AND WITH INVESTMENTS IN A BOND AND SEVERAL STOCKS
}

UDC 519.21

M. O. ANDROSHCHUK

\begin{abstract}
We consider a risk process generalizing the classical Cramér-Lundberg process. The feature of the process is that its price function depends on the current reserve of an insurance company as well as on its portfolio consisting of a riskless bond and a finite number of risky assets, modeled by geometric Brownian motions. We obtain an analogue of the classical exponential estimate for the ruin probability in this case. It turns out that the estimate for the model with investments is better than the corresponding estimate for the classical model without investments.
\end{abstract}

\section{INTRODUCTION}

Since 1903, when Lundberg introduced a collective risk model, the estimation of ruin probabilities of insurance companies has been the main topic in risk theory. Several authors provide various generalizations of the classical risk model. Sometimes the authors include inflation in the model and solve the problem of the estimation of the current value of the money (see [1]-3]). The claims are sometimes modeled by a more general point process than the Poisson process, say by the renewal process or by the Cox process (see [4, 5, 2]). In 6], the classical risk process contains a stochastic return on investments, modeled by Brownian motion.

Useful from the point of view of applications are the risk models where an insurance company is allowed to buy bonds and stocks in a stock market (see [1, 7]). Another generalization of the classical model, which we will use in this paper, is due to the dependence of the insurance premiums on the current reserve of the company at every instance. It is natural to assume that an insurer asks for lower premiums if it has a large reserve and vice versa (see [8, 7]).

\section{THE MODEL}

In the current paper, we consider the problem of estimation of the ruin probability for an insurance company in the case where the price function depends on the current reserve and the company is allowed to buy a riskless bond and risky asset in a stock market. Our setting generalizes the model studied in the paper [7] in the sense that we allow the insurance company to buy not only a single stock but a finite number of stocks as well as a riskless bond, that is, if we allow the company to diverse its capital.

2000 Mathematics Subject Classification. Primary 60H05; Secondary 60G15.

Key words and phrases. Semimartingale, local martingale, ruin probability, investment strategy. 
The risk process is described by the following equation (the integrals are understood in a formal way):

$$
\begin{array}{r}
R_{t}(u, \bar{K})=u-\sum_{k=1}^{\mathbf{N}_{t}} U_{k}+\int_{0}^{t} p\left(R_{s}\right) d s+\sum_{i=1}^{n} \int_{0}^{t} \frac{K_{s}^{i}}{S_{s}^{i}} d S_{s}^{i}+\int_{0}^{t} \frac{R_{s}-\sum_{i=1}^{n} K_{s}^{i}}{e^{\delta s}} d e^{\delta s} \\
t \geq 0
\end{array}
$$

where $u>0$ is the initial capital of an insurance company, $\left\{\mathbf{N}_{t}, t \geq 0\right\}$ is the Poisson process modelling the flow of insurance claims and which has a constant intensity $\beta>0$, $\left\{U_{k}, k \geq 1\right\}$ is a sequence of nonnegative identically distributed random variables meaning the insurance payments according to claims (this sequence is assumed to be independent of the stochastic process $\left.\left\{\mathbf{N}_{t}, t \geq 0\right\}\right), p\left(R_{s}\right)$ is the process of the insurance premiums (price function) depending on the current reserve of the company at a moment $s, K_{s}^{i}$ is the amount of money invested by the insurer in a stock $i, i=1, \ldots, n$; the rest of the money $R_{s}-\sum_{i=1}^{n} K_{s}^{i}$ is invested in a riskless bond (deposited in a bank account) with a constant rate $\delta$.

The evolution of the price of stocks is described by the geometrical Brownian motions

$$
d S_{t}^{i}=S_{t}^{i}\left(a_{i} d t+b_{i} d W_{t}^{i}\right)
$$

where $a_{i}>0$ and $b_{i}>0$ are fixed constants and where $\left\{W_{t}^{i}, t \geq 0\right\}$ are standard Brownian motions being independent of the compound Poisson process and such that $\operatorname{Cov}\left(W_{t}^{i}, W_{t}^{j}\right)=\rho_{i j} t, i=1, \ldots, n, j=1, \ldots, n$. Note that the covariance coefficients $\rho_{i j}$ are such that $\rho_{i j}=\rho_{j i}$ and $\rho_{i i}=1$.

We assume that the $\sigma$-algebras generated by the processes $\left\{W_{t}^{i}, t \geq 0, i=1, \ldots, n\right\}$, $\left\{\mathbf{N}_{t}, t \geq 0\right\}$, and the sequence of random variables $\left\{U_{k}, k \geq 1\right\}$ are independent.

\section{The SUPERMARTINGALE PROPERTy OF THE EXPONENTIAL PROCESS}

Denote by $\mathbb{F}=\left(\mathcal{F}_{t}\right)_{t \geq 0}$ the filtration generated by the compound Poisson process $\left\{\sum_{k=1}^{\mathbf{N}_{t}} U_{k}, t \geq 0\right\}$ and processes $\left\{W_{t}^{i}, t \geq 0, i=1, \ldots, n\right\}$. In what follows the symbol $E_{t}[\cdot]$ stands for the conditional expectation $\mathrm{E}\left[\cdot \mid \mathcal{F}_{t}\right]$.

We assume that the function $p(x)$ and strategies $K_{s}^{i}, s \geq 0, i=1, \ldots, n$, are such that equation (1) has a solution in an arbitrary interval $[0, T]$. Sufficient conditions for the existence of a solution are discussed in Remark 5 below.

We rewrite equation (1) in the following form:

$$
\begin{aligned}
R_{t}(u, \bar{K})= & u-\sum_{k=1}^{\mathbf{N}_{t}} U_{k}+\int_{0}^{t} p\left(R_{s}\right) d s+\sum_{i=1}^{n} a_{i} \int_{0}^{t} K_{s}^{i} d s \\
& +\delta \int_{0}^{t}\left(R_{s}-\sum_{i=1}^{n} K_{s}^{i}\right) d s+\sum_{i=1}^{n} b_{i} \int_{0}^{t} K_{s}^{i} d W_{s}^{i}, \quad t \geq 0 .
\end{aligned}
$$

All the terms in equation (3) are well defined if the strategy $\bar{K}_{t}$ belongs to the set of admissible strategies, that is, if the strategy $\bar{K}_{t}$ satisfies the following conditions (K1) and (K2):

(K1) the vector process $\bar{K}_{t}=\left\{K_{t}^{1}, \ldots, K_{t}^{n}\right\}$ is predictable with respect to the $\sigma$ algebra $\mathcal{F}_{t}$

(K2) $\mathrm{P}\left\{\int_{0}^{t}\left(K_{s}^{i}\right)^{2} d s<\infty, i=1, \ldots, n\right\}=1, t \geq 0$.

In order to prove that a solution of equation (11) exists we assume that

$(\mathbf{P 1}) p(\cdot): \mathbb{R} \rightarrow \mathbb{R}_{+}$is a measurable nonnegative function. 
We introduce the following notation: $\tau(u, \bar{K}):=\inf \left\{t \geqslant 0: R_{t}(u, \bar{K}) \leqslant 0\right\}$ is the ruin moment of an insurance company with an initial reserve $u$ and investment strategy $\bar{K}$. We assume that $\tau(u, \bar{K})=+\infty$ if $R_{t}>0$ for all $t>0$.

In what follows we assume that conditions (K1), (K2), and (P1) hold.

Denote by $h: \mathbb{R}_{+} \rightarrow \mathbb{R}_{+}$the shifted moment generating function for the random variables $U_{k}, k \geq 1$, describing the insurance payments. The shift is chosen such that $h(0)=0$; namely,

$$
h(r)=\mathrm{E}\left[e^{r U}\right]-1
$$

We make a classical assumption concerning the function $h(r)$; namely we assume that there exists a number $r_{\infty} \in(0, \infty]$ such that $h(r)<\infty$ for all $r<r_{\infty}$ and $h(r) \rightarrow \infty$ as $r \uparrow r_{\infty}$. The function $h(r)$ is increasing, concave, and continuous on $\left[0, r_{\infty}\right)$ (see [4]).

Next we introduce the process

$$
X_{t}(u, \bar{K}, r):=e^{-r R_{t}(u, \bar{K})}, \quad r \in[0,+\infty) .
$$

Substituting $R_{t}$ defined by (3) into the right hand side of the latter equality, we get

$$
\begin{aligned}
X_{t}(u, \bar{K}, r)= & e^{-r u} \cdot \exp \left(-r\left(-\sum_{k=1}^{\mathbf{N}_{t}} U_{k}+\int_{0}^{t} p\left(R_{s}\right) d s+\sum_{i=1}^{n} a_{i} \int_{0}^{t} K_{s}^{i} d s\right)\right) \\
& \times \exp \left(-r\left(\delta \int_{0}^{t}\left(R_{s}-\sum_{i=1}^{n} K_{s}^{i}\right) d s+\sum_{i=1}^{n} b_{i} \int_{0}^{t} K_{s}^{i} d W_{s}^{i}\right)\right) .
\end{aligned}
$$

Theorem 1. Let conditions (P1), (K1), and (K2) hold for the model described by equality (10) where $r>0$ is some number, $\bar{K}_{t}$ is an investment strategy, and $p(x)$ is the price function. We also assume that

(R1) $\mathrm{P}\left\{\int_{0+}^{t} e^{-r R_{s-}}\left|R_{s}\right| d s<\infty\right\}=1, t \geq 0$,

(R2) $\mathrm{P}\left\{\int_{0+}^{t} e^{-2 r R_{s-}}\left(K_{s}^{i}\right)^{2} d s<\infty, i=1, \ldots, n\right\}=1, t \geq 0$,

(R3) $\mathrm{P}\left\{\int_{0+}^{t} e^{-r R_{s-}} d s<\infty\right\}=1, t \geq 0$,

(K3)

$$
\begin{aligned}
\mathrm{P}\left\{r^{2}\left(\frac{1}{2} \sum_{i=1}^{n} b_{i}^{2}\left(K_{t}^{i}\right)^{2}+\frac{1}{2} \sum_{i=1}^{n} \sum_{\substack{k=1, \ldots, n \\
k \neq i}} b_{i} b_{k} K_{t}^{i} K_{t}^{k} \rho_{i k}\right)\right. \\
\left.\quad-r\left(p\left(R_{t}\right)+\delta R_{t}+\sum_{i=1}^{n}\left(a_{i}-\delta\right) K_{t}^{i}\right)+\beta h(r) \leq 0, t \geq 0\right\}=1 .
\end{aligned}
$$

Then the process $\left\{X_{t}(u, \bar{K}, r), t \geq 0\right\}$ is a supermartingale with respect to the flow of $\sigma$-algebras $\mathcal{F}_{t}$. If

$$
\begin{aligned}
\mathrm{P}\left\{r^{2}\left(\frac{1}{2} \sum_{i=1}^{n} b_{i}^{2}\left(K_{t}^{i}\right)^{2}+\frac{1}{2} \sum_{i=1}^{n} \sum_{\substack{k=1, \ldots, n \\
k \neq i}} b_{i} b_{k} K_{t}^{i} K_{t}^{k} \rho_{i k}\right)\right. \\
\left.\quad-r\left(p\left(R_{t}\right)+\delta R_{t}+\sum_{i=1}^{n}\left(a_{i}-\delta\right) K_{t}^{i}\right)+\beta h(r)=0, t \geq 0\right\}=1,
\end{aligned}
$$

then the process $\left\{X_{t}(u, \bar{K}, r), t \geq 0\right\}$ is a local martingale with respect to $\mathcal{F}_{t}$. 
Proof. Interchanging the terms in (5) we get

$$
\begin{aligned}
X_{t}(u, \bar{K}, r)= & e^{-r u} \cdot \exp \left(r \sum_{k=1}^{\mathbf{N}_{t}} U_{k}\right) \\
& \times \exp \left(-r \int_{0}^{t}\left(p\left(R_{s}\right)+\sum_{i=1}^{n} a_{i} K_{s}^{i}+\delta\left(R_{s}-\sum_{i=1}^{n} K_{s}^{i}\right)\right) d s\right) \\
& \times \exp \left(-r \sum_{i=1}^{n} b_{i} \int_{0}^{t} K_{s}^{i} d W_{s}^{i}\right) .
\end{aligned}
$$

Now we represent the process $\left\{X_{t}(u, \bar{K}, r), t \geq 0\right\}$ in the form of an exponent:

$$
X_{t}(u, \bar{K}, r)=e^{-r R_{t}(u, \bar{K})}:=e^{Y_{t}(u, \bar{K}, r)}, \quad t \geq 0,
$$

where

$$
\begin{aligned}
Y_{t}(u, \bar{K}, r) & =Y_{0}+r \cdot \sum_{k=1}^{\mathbf{N}_{t}} U_{k}+\int_{0}^{t} f(s) d s+\sum_{i=1}^{n} \int_{0}^{t} g_{i}(s) d W_{s}^{i}, \\
Y_{0} & :=-r u, \\
f(s): & =-r\left(p\left(R_{s}\right)+\sum_{i=1}^{n} a_{i} K_{s}^{i}+\delta\left(R_{s}-\sum_{i=1}^{n} K_{s}^{i}\right)\right), \\
g_{i}(s): & =-r b_{i} K_{s}^{i} .
\end{aligned}
$$

Since the process $R_{t}$, as a sum of local martingales and processes of bounded variation, is a semimartingale, the process $X_{t}(u, \bar{K}, r)=\exp \left\{-r R_{t}(u, \bar{K}, r)\right\}$ is a semimartingale in view of Itô's formula applied to the exponential function $f(x)=e^{-r x}$. Note that the processes $\int_{0}^{t} K_{i}^{s} d W_{s}^{i}$ are square integrable martingales by condition (K2), the process

$$
\sum_{k=1}^{\mathbf{N}_{t}} U_{k}-\beta t \mathrm{E} U_{1}
$$

is a martingale as a compensated process with independent increments, and $\int_{0}^{t} p\left(R_{s}\right) d s$, $\int_{0}^{t} K_{s}^{i} d s$, and $\int_{0}^{t}\left(R_{s}-\sum_{i=1}^{n} K_{s}^{i}\right) d s$ are stochastic processes of bounded variation.

The Itô formula can be written for semimartingale processes in the following form:

$$
\begin{aligned}
F\left(Y_{t}\right)-F\left(Y_{0}\right)= & \int_{0_{+}}^{t} F^{\prime}\left(Y_{s-}\right) d Y_{s}+\frac{1}{2} \int_{0_{+}}^{t} F^{\prime \prime}\left(Y_{s-}\right) d\langle Y, Y\rangle_{s}^{c} \\
& +\sum_{0<s \leq t}\left(F\left(Y_{s}\right)-F\left(Y_{s-}\right)-F^{\prime}\left(Y_{s-}\right)\left(Y_{s}-Y_{s-}\right)\right),
\end{aligned}
$$

where $\left\{Y_{t}, t \geq 0\right\}$ is a semimartingale and $F \in \mathcal{C}^{2}(\mathbb{R})$ (see [9, pp. 78-79]).

Substituting $F(y)=F^{\prime}(y)=F^{\prime \prime}(y)=e^{y}$ to (7) we obtain

$$
\begin{aligned}
e^{Y_{t}}= & e^{Y_{0}}+\int_{0_{+}}^{t} e^{Y_{s-}} d Y_{s}+\frac{1}{2} \int_{0_{+}}^{t} e^{Y_{s-}} d\langle Y, Y\rangle_{s}^{c} \\
& +\sum_{0<s \leq t}\left(e^{Y_{s}}-e^{Y_{s-}}-e^{Y_{s-}}\left(Y_{s}-Y_{s-}\right)\right),
\end{aligned}
$$


where

$$
\begin{gathered}
Y_{t-}(u, \bar{K}, r)=Y_{0}+r \cdot \sum_{k=1}^{\mathbf{N}_{t-}} U_{k}+\int_{0}^{t} f(s) d s+\sum_{i=1}^{n} \int_{0}^{t} g_{i}(s) d W_{s}^{i}, \\
d Y_{s}=r d\left(\sum_{k=1}^{\mathbf{N}_{s}} U_{k}\right)+f(s) d s+\sum_{i=1}^{n} g_{i}(s) d W_{s}^{i}, \\
Y_{s}-Y_{s-}=r U_{\mathbf{N}_{s}} I\left\{\triangle \mathbf{N}_{s} \neq 0\right\}, \\
e^{Y_{s}}-e^{Y_{s-}}=e^{Y_{s-}}\left(e^{r U_{\mathbf{N}_{s}} I\left\{\triangle \mathbf{N}_{s} \neq 0\right\}}-1\right), \\
d\langle Y, Y\rangle_{s}^{c}=\sum_{i=1}^{n} g_{i}^{2}(s) d s+\sum_{i=1}^{n} \sum_{\substack{k=1, \ldots, n \\
k \neq i}} g_{i}(s) g_{k}(s) \rho_{i k} d s .
\end{gathered}
$$

Taking into account (91)-(13) we rewrite relation (8) as follows:

$$
\begin{aligned}
X_{t}=e^{Y_{t}}= & e^{Y_{0}}+r \int_{0_{+}}^{t} e^{Y_{s-}} d\left(\sum_{k=1}^{\mathbf{N}_{t}} U_{k}\right)+\int_{0_{+}}^{t} e^{Y_{s-}} f(s) d s \\
& +\sum_{i=1}^{n} \int_{0_{+}}^{t} e^{Y_{s-}} g_{i}(s) d W_{s}^{i} \\
& +\frac{1}{2} \int_{0_{+}}^{t} e^{Y_{s-}}\left(\sum_{i=1}^{n} g_{i}^{2}(s)+\sum_{i=1}^{n} \sum_{\substack{k=1, \ldots, n \\
k \neq i}} g_{i}(s) g_{k}(s) \rho_{i k}\right) d s \\
& +\sum_{0<s \leq t} e^{Y_{s-}}\left(e^{r U_{\mathbf{N}_{s}} I\left\{\triangle \mathbf{N}_{s} \neq 0\right\}}-1-r U_{\mathbf{N}_{s}} I\left\{\triangle \mathbf{N}_{s} \neq 0\right\}\right) .
\end{aligned}
$$

The terms

$$
r \int_{0_{+}}^{t} e^{Y_{s-}} d\left(\sum_{k=1}^{\mathbf{N}_{t}} U_{k}\right) \quad \text { and } \quad\left(-\sum_{0<s \leq t} e^{Y_{s-}} r U_{\mathbf{N}_{s}} I\left\{\triangle \mathbf{N}_{s} \neq 0\right\}\right)
$$

cancel each other in (14).

Note that the integrals $\int_{0_{+}}^{t} e^{Y_{s-}} g_{i}(s) d W_{s}^{i}, i=1, \ldots, n$, are well defined in view of condition (R2).

The processes $M_{t}^{i}:=\int_{0_{+}}^{t} e^{Y_{s-}} g_{i}(s) d W_{s}^{i}, i=1, \ldots, n$, are square integrable local martingales with Markov moments

$$
\tau_{N}^{i}:=\inf \left\{t \geq 0: \int_{0}^{t}\left(e^{Y_{s-}} g_{i}(s)\right)^{2} d s \geq N\right\} \wedge N, \quad N \geq 1, i=1, \ldots, n .
$$

Explanation. Condition (R2) implies

$$
\left.\mathrm{P}\left\{\tau_{N}^{i}<T\right\} \leq \mathrm{P}\left\{\int_{0}^{T}\left(e^{Y_{s-}} g_{i}(s)\right)^{2} d s \geq N\right)\right\}
$$

for $N>T$ and the right hand side tends to zero as $N \rightarrow \infty$. In other words, the moments $\left\{\tau_{N}^{i}, N \geq 1\right\}_{i=1}^{n}$ are Markovian and such that $\mathrm{P}\left\{\tau_{N}^{i} \leq N\right\}=1, \mathrm{P}\left\{\tau_{N}^{i} \leq \tau_{N+1}^{i}\right\}=1$, and $\tau_{N}^{i} \uparrow \infty$ almost surely. Moreover, the processes $\int_{0_{+}}^{t \wedge \tau_{N}^{i}} e^{Y_{s-}} g_{i}(s) d W_{s}^{i}, i=1, \ldots, n$, are square integrable martingales. 
The process

$$
P_{t}:=\sum_{0<s \leq t} e^{Y_{s-}}\left(e^{r U_{\mathbf{N}_{s}} I\left\{\Delta \mathbf{N}_{s} \neq 0\right\}}-1\right)
$$

is nondecreasing. We rewrite it in the integral form:

$$
P_{t}=\int_{0}^{t} e^{Y_{s-}} d O_{s}
$$

where

$$
O_{t}:=\sum_{0<s \leq t}\left(e^{r U_{\mathbf{N}_{s}} I\left\{\triangle \mathbf{N}_{s} \neq 0\right\}}-1\right), \quad t \geq 0 .
$$

Using the Wald identity we prove for all $t \geq 0$ that

$$
\mathrm{E} \sum_{0<s \leq t}\left(e^{r U_{\mathbf{N}_{s}} I\left\{\triangle \mathbf{N}_{s} \neq 0\right\}}-1\right)=\mathrm{E} \sum_{k=1}^{\mathbf{N}_{t}}\left(e^{r U_{k}}-1\right)=\beta \operatorname{th}(r) \Rightarrow \mathrm{E} O_{t}<\infty .
$$

Moreover $\left\{O_{t}, t \geq 0\right\}$ is a process with independent increments; thus the compensated process $\left\{O_{t}-\mathrm{E} O_{t}, t \geq 0\right\}$ is a martingale.

The sequence

$$
\sigma_{N}:=\inf \left\{t \geq 0: Y_{t} \geq N\right\} \wedge N, \quad N \geq 1,
$$

of stopping times is such that

$$
\begin{gathered}
\mathrm{P}\left\{\sigma_{N} \leq N\right\}=1, \\
\mathrm{P}\left\{\sigma_{N} \leq \sigma_{N+1}\right\}=1, \\
\sigma_{N} \uparrow \infty \text { almost surely, }
\end{gathered}
$$

since

$$
\mathrm{P}\left\{\sigma_{N}<T\right\} \leq \mathrm{P}\left\{\sup \left\{t \in[0, T]:\left(-r R_{t}\right) \geq N\right\}\right\} \underset{N \rightarrow \infty}{\longmapsto} 0 .
$$

The latter relation follows from the definition of the process $\left\{R_{t}, t \geq 0\right\}$. Note that this process may attain negative values resulting from payments due to insurance payments $\left\{U_{k}, k \geq 1\right\}$; however, only a finite number of claims occur during a finite time and the total amount of the payments is finite with probability 1 .

Thus the process

$$
P_{t}-\beta h(r) \int_{0_{+}}^{t} e^{Y_{s-}} d s
$$

is a local martingale, since

$$
\int_{0}^{t \wedge \sigma_{N}} e^{Y_{s-}} d\left(O_{s}-\mathrm{E} O_{s}\right)=P_{t \wedge \sigma_{N}}-\beta h(r) \int_{0_{+}}^{t \wedge \sigma_{N}} e^{Y_{s-}} d s
$$

is a martingale.

Using properties of local martingales (see [9, p. 37]) we prove that the process

$$
\sum_{i=1}^{n} M_{t}^{i}+P_{t}-\beta h(r) \int_{0_{+}}^{t} e^{Y_{s-}} d s
$$

also is a local martingale whose fundamental sequence is $\tau_{N} \wedge \sigma_{N}, N \geq 1$.

Consider the difference

$$
V_{t}:=X_{t}-X_{0}-\sum_{i=1}^{n} M_{t}^{i}-\left(P_{t}-\beta h(r) \int_{0_{+}}^{t} e^{Y_{s-}} d s\right) .
$$


Substituting the corresponding expressions for the terms on the right hand side we get

$$
\begin{aligned}
V_{t}= & \int_{0_{+}}^{t} e^{Y_{s-}}\left(f(s)+\frac{1}{2} \sum_{i=1}^{n} g_{i}^{2}(s)+\frac{1}{2} \sum_{i=1}^{n} \sum_{\substack{k=1, \ldots, n \\
k \neq i}} g_{i}(s) g_{k}(s) \rho_{i k}+\beta h(r)\right) d s \\
= & \int_{0_{+}}^{t} e^{-r R_{s-}}\left(-r\left(p\left(R_{s}\right)+\sum_{i=1}^{n} a_{i} K_{s}^{i}+\delta\left(R_{s}-\sum_{i=1}^{n} K_{s}^{i}\right)\right)+\beta h(r)\right) d s \\
& +\int_{0_{+}}^{t} e^{-r R_{s-}}\left(\frac{1}{2} \sum_{i=1}^{n} r^{2} b_{i}^{2}\left(K_{s}^{i}\right)^{2}+\frac{1}{2} \sum_{i=1}^{n} \sum_{\substack{k=1, \ldots, n \\
k \neq i}} r^{2} b_{i} b_{k} K_{s}^{i} K_{s}^{k} \rho_{i k}\right) d s .
\end{aligned}
$$

The Lebesgue integral on the right hand side of (15) is well defined by conditions (R1)(R3) and

$$
\mathrm{P}\left\{\int_{0+}^{t} e^{-r R_{s-}}\left(K_{s}^{i}\right)^{2} d s<\infty, i=1, \ldots, n\right\}=1, \quad t \geq 0 .
$$

In its turn, the latter equality follows from (K2) and (R2).

If

$$
\left\{V_{t \wedge \tau_{N} \wedge \sigma_{N}}, t \geq 0, N \geq 1\right\}
$$

is a predictable decreasing process, then the Doob-Meyer decomposition (see, for example, [4. p. 105]) implies that $\left\{X_{t}, t \geq 0\right\}$ is a local semimartingale. It is obvious that the process $V_{t}$ is predictable, since it is continuous in $t$. It remains to find conditions under which the process

$$
\left\{V_{t \wedge \tau_{N} \wedge \sigma_{N}}, t \geq 0, N \geq 1\right\}
$$

is decreasing.

The property

$$
\begin{aligned}
V_{T}-V_{t}= & \int_{t}^{T} e^{-r R_{s-}}\left(-r\left(p\left(R_{s}\right)+\sum_{i=1}^{n} a_{i} K_{s}^{i}+\delta\left(R_{s}-\sum_{i=1}^{n} K_{s}^{i}\right)\right)+\beta h(r)\right) d s \\
& +\int_{t}^{T} e^{-r R_{s-}}\left(\frac{1}{2} \sum_{i=1}^{n} r^{2} b_{i}^{2}\left(K_{s}^{i}\right)^{2}+\frac{1}{2} \sum_{i=1}^{n} \sum_{\substack{k=1, \ldots, n \\
k \neq i}} r^{2} b_{i} b_{k} K_{s}^{i} K_{s}^{k} \rho_{i k}\right) d s \\
\leq & 0
\end{aligned}
$$

holds for all $T \geq 0$ and $t \in[0, T]$ in view of condition (K3).

Thus

$$
X_{t}=X_{0}+M_{t}+P_{t}-\beta h(r) \int_{0_{+}}^{t} e^{Y_{s-}} d s+V_{t}
$$

is a nonnegative local supermartingale. Now Fatou's lemma implies that $X_{t}$ is a supermartingale with respect to the flow of $\sigma$-algebras $\mathcal{F}_{t}$.

The theorem is proved.

Remark 1. Assume that $t \wedge \tau(u)$ is substituted for the upper limit of integration in conditions (K2), (R1), (R2), and (R3). If condition (K3) holds for all $t \in[0, \tau(u)]$, then one can prove similarly to Theorem 1 that $\left\{X_{t \wedge \tau(u)}, t \geq 0\right\}$ is a semimartingale with respect to the flow of $\sigma$-algebras $\mathcal{F}_{t}$. 


\section{An estimate of the RUin PRobability}

Now we minimize the functional in condition (K3),

$$
\begin{aligned}
F\left(\bar{K}_{s}\right):= & \frac{r^{2}}{2}\left(\sum_{i=1}^{n} b_{i}^{2}\left(K_{s}^{i}\right)^{2}+\sum_{i=1}^{n} \sum_{\substack{k=1, \ldots, n \\
k \neq i}} b_{i} b_{k} K_{s}^{i} K_{s}^{k} \rho_{i k}\right) \\
& -r\left(p\left(R_{s}\right)+\delta R_{s}+\sum_{i=1}^{n}\left(a_{i}-\delta\right) K_{s}^{i}\right)+\beta h(r),
\end{aligned}
$$

over admissible strategies $K_{s}^{i} \in \mathbb{R}, i=1, \ldots, n$.

It is clear that the point

$$
\widehat{K}_{s}^{i}=\frac{a_{i}-\delta}{r b_{i}^{2}}-\frac{1}{2 b_{i}} \sum_{\substack{k=1, \ldots, n \\ k \neq i}} b_{k} \widehat{K}_{s}^{k} \rho_{i k}, \quad i=1, \ldots, n
$$

is a minimum point of functional (16).

We find the value of functional (16) at this point:

$$
\begin{aligned}
F\left(\widehat{\widehat{K}}_{s}\right)= & \frac{r^{2}}{2} \sum_{i=1}^{n} b_{i}^{2}\left(\frac{a_{i}-\delta}{r b_{i}^{2}}-\frac{1}{2 b_{i}} \sum_{\substack{k=1, \ldots, n \\
k \neq i}} b_{k} \widehat{K}_{s}^{k} \rho_{i k}\right)^{2} \\
& +\frac{r^{2}}{2} \sum_{i=1}^{n} b_{i}\left(\frac{a_{i}-\delta}{r b_{i}^{2}}-\frac{1}{2 b_{i}} \sum_{\substack{k=1, \ldots, n \\
k \neq i}} b_{k} \widehat{K}_{s}^{k} \rho_{i k}\right) \sum_{\substack{k=1, \ldots, n \\
k \neq i}} b_{k} \widehat{K}_{s}^{k} \rho_{i k} \\
& -r \sum_{i=1}^{n}\left(a_{i}-\delta\right)\left(\frac{a_{i}-\delta}{r b_{i}^{2}}-\frac{1}{2 b_{i}} \underset{\substack{k=1, \ldots, n \\
k \neq i}}{\sum_{k}} b_{k} \widehat{K}_{s}^{k} \rho_{i k}\right)-r p\left(R_{s}\right)-r \delta R_{s}+\beta h(r) .
\end{aligned}
$$

After some algebra we get

$$
\begin{aligned}
F\left(\widehat{\widehat{K}}_{s}\right)= & -\frac{1}{2} \sum_{i=1}^{n} \frac{\left(a_{i}-\delta\right)^{2}}{b_{i}^{2}}-\frac{r^{2}}{8} \sum_{i=1}^{n}\left(\sum_{\substack{k=1, \ldots, n \\
k \neq i}} b_{k} \widehat{K}_{s}^{k} \rho_{i k}\right)^{2} \\
& +\frac{r}{2} \sum_{i=1}^{n} \frac{\left(a_{i}-\delta\right)}{b_{i}}\left(\sum_{\substack{k=1, \ldots, n \\
k \neq i}} b_{k} \widehat{K}_{s}^{k} \rho_{i k}\right)-r p\left(R_{s}\right)-r \delta R_{s}+\beta h(r) .
\end{aligned}
$$

Let

$$
\Phi_{s}^{i}:=\sum_{\substack{k=1, \ldots, n \\ k \neq i}} b_{k} \widehat{K}_{s}^{k} \rho_{i k}
$$


Then equality (18) can be rewritten as follows:

$$
\begin{aligned}
F\left(\overline{\widehat{K}}_{s}\right)= & \widetilde{F}\left(\bar{\Phi}_{s}\right) \\
= & -\frac{1}{2} \sum_{i=1}^{n} \frac{\left(a_{i}-\delta\right)^{2}}{b_{i}^{2}}-\frac{r^{2}}{8} \sum_{i=1}^{n}\left(\Phi_{s}^{i}\right)^{2}+\frac{r}{2} \sum_{i=1}^{n} \frac{\left(a_{i}-\delta\right)}{b_{i}} \Phi_{s}^{i}-r p\left(R_{s}\right)-r \delta R_{s}+\beta h(r) \\
= & -\frac{1}{8}\left(r^{2} \sum_{i=1}^{n}\left(\Phi_{s}^{i}\right)^{2}-4 r \sum_{i=1}^{n} \frac{\left(a_{i}-\delta\right)}{b_{i}} \Phi_{s}^{i}+4 \sum_{i=1}^{n} \frac{\left(a_{i}-\delta\right)^{2}}{b_{i}^{2}}\right) \\
& -r p\left(R_{s}\right)-r \delta R_{s}+\beta h(r) \\
= & -\frac{1}{8} \sum_{i=1}^{n}\left(r \Phi_{s}^{i}-2 \frac{a_{i}-\delta}{b_{i}}\right)^{2}-r p\left(R_{s}\right)-r \delta R_{s}+\beta h(r) .
\end{aligned}
$$

The inequality $F\left(\widehat{\widehat{K}}_{s}\right) \leq 0$ holds if

$$
\beta h(r) \leq \frac{1}{8} \sum_{i=1}^{n}\left(r \Phi_{s}^{i}-2 \frac{a_{i}-\delta}{b_{i}}\right)^{2}+r p\left(R_{s}\right)+r \delta R_{s} .
$$

Considering system (17) we obtain

$$
\Phi_{s}^{i}=\sum_{\substack{k=1, \ldots, n \\ k \neq i}} b_{k} \widehat{K}_{s}^{k} \rho_{i k}=2 b_{i}\left(\frac{a_{i}-\delta}{r b_{i}^{2}}-\widehat{K}_{s}^{i}\right) .
$$

Thus relation (19) can be rewritten as follows:

$$
\begin{aligned}
\beta h(r) & \leq \frac{1}{8} \sum_{i=1}^{n}\left(r \cdot 2 b_{i}\left(\frac{a_{i}-\delta}{r b_{i}^{2}}-\widehat{K}_{s}^{i}\right)-2 \frac{a_{i}-\delta}{b_{i}}\right)^{2}+r p\left(R_{s}\right)+r \delta R_{s} \\
& \Longleftrightarrow \beta h(r) \leq \sum_{i=1}^{n} \frac{r^{2} b_{i}^{2}\left(\widehat{K}_{s}^{i}\right)^{2}}{2}+r p\left(R_{s}\right)+r \delta R_{s} .
\end{aligned}
$$

One can use the matrix form for the system of equations (17):

$$
\left(\begin{array}{ccccc}
1 & \frac{b_{2}}{2 b_{1}} \rho_{12} & \frac{b_{3}}{2 b_{1}} \rho_{13} & \ldots & \frac{b_{n}}{2 b_{1}} \rho_{1 n} \\
\frac{b_{1}}{2 b_{2}} \rho_{21} & 1 & \frac{b_{3}}{2 b_{2}} \rho_{23} & \ldots & \frac{b_{n}}{2 b_{2}} \rho_{2 n} \\
\vdots & \vdots & \vdots & \ddots & \vdots \\
\frac{b_{1}}{2 b_{n}} \rho_{n 1} & \frac{b_{2}}{2 b_{n}} \rho_{n 2} & \frac{b_{3}}{2 b_{n}} \rho_{n 3} & \ldots & 1
\end{array}\right) \cdot \bar{K}_{s}=\left(\begin{array}{c}
\frac{a_{1}-\delta}{r b b_{1}^{2}} \\
\frac{a_{2}-\delta}{r b_{2}^{2}} \\
\vdots \\
\frac{a_{n}-\delta}{r b_{n}^{2}}
\end{array}\right)
$$

Remark 2. The solution of system (21) can easily be found by Cramer's rule if

$$
\rho_{i k}=\rho_{i} \cdot \rho_{k}, \quad i=1, \ldots, n, k=1, \ldots, n,
$$

and

$$
\rho_{i} \neq \pm \sqrt{2}, \quad i=1, \ldots, n
$$

In this case,

$$
\left|\begin{array}{ccccc}
1 & \frac{b_{2}}{2 b_{1}} \rho_{1} \rho_{2} & \frac{b_{3}}{2 b_{1}} \rho_{1} \rho_{3} & \ldots & \frac{b_{n}}{2 b_{1}} \rho_{1} \rho_{n} \\
\frac{b_{1}}{2 b_{2}} \rho_{2} \rho_{1} & 1 & \frac{b_{3}}{2 b_{2}} \rho_{2} \rho_{3} & \ldots & \frac{b_{n}}{2 b_{2}} \rho_{2} \rho_{n} \\
\vdots & \vdots & \vdots & \ddots & \vdots \\
\frac{b_{1}}{2 b_{n}} \rho_{n} \rho_{1} & \frac{b_{2}}{2 b_{n}} \rho_{n} \rho_{2} & \frac{b_{3}}{2 b_{n}} \rho_{n} \rho_{3} & \ldots & 1
\end{array}\right|=\prod_{i=1}^{n}\left(1-\frac{\rho_{i}^{2}}{2}\right) \cdot\left(1+\sum_{j=1}^{n} \frac{\rho_{j}^{2}}{2-\rho_{j}^{2}}\right)
$$


(see [10, Problem 227]), whence we have found the solution of system (21):

$$
\widehat{K}_{s}^{i}=\frac{\left|\begin{array}{ccccccccc}
1 & \frac{b_{2}}{2 b_{1}} \rho_{1} \rho_{2} & \frac{b_{3}}{2 b_{1}} \rho_{1} \rho_{3} & \ldots & \frac{b_{i-1}}{2 b_{1}} \rho_{1} \rho_{i-1} & \frac{a_{1}-\delta}{r b_{1}^{2}} & \frac{b_{i+1}}{2 b_{1}} \rho_{1} \rho_{i+1} & \ldots & \frac{b_{n}}{2 b_{1}} \rho_{1} \rho_{n} \\
\frac{b_{1}}{2 b_{2}} \rho_{2} \rho_{1} & 1 & \frac{b_{3}}{2 b_{2}} \rho_{2} \rho_{3} & \ldots & \frac{b_{i-1}}{2 b_{2}} \rho_{2} \rho_{i-1} & \frac{a_{2}-\delta}{r b_{2}^{2}} & \frac{b_{i+1}}{2 b_{2}} \rho_{2} \rho_{i+1} & \ldots & \frac{b_{n}}{2 b_{2}} \rho_{2} \rho_{n} \\
\vdots & \vdots & \vdots & \ddots & \vdots & \vdots & \vdots & \ddots & \vdots \\
\frac{b_{1}}{2 b_{n}} \rho_{n} \rho_{1} & \frac{b_{2}}{2 b_{n}} \rho_{n} \rho_{2} & \frac{b_{3}}{2 b_{n}} \rho_{n} \rho_{3} & \ldots & \frac{b_{i-1}}{2 b_{n}} \rho_{n} \rho_{i-1} & \frac{a_{n}-\delta}{r b_{n}^{2}} & \frac{b_{i+1}}{2 b_{n}} \rho_{n} \rho_{i+1} & \ldots & 1
\end{array}\right|}{\prod_{i=1}^{n}\left(1-\frac{\rho_{i}^{2}}{2}\right) \cdot\left(1+\sum_{j=1}^{n} \frac{\rho_{j}^{2}}{2-\rho_{j}^{2}}\right)}
$$

$i=1, \ldots, n, s \geq 0$.

In the general case,

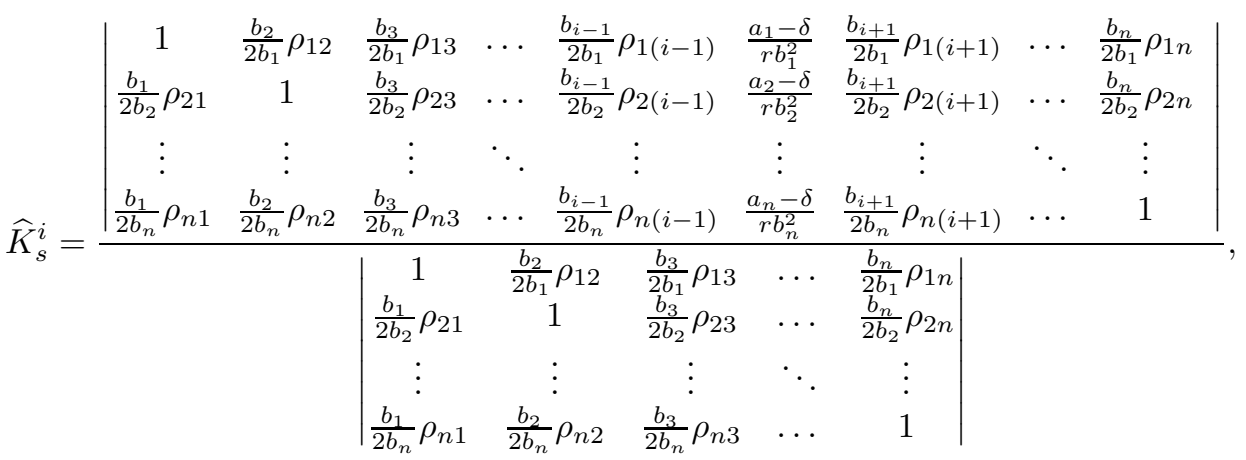

$i=1, \ldots, n, s \geq 0$.

Theorem 2. Let condition (P1) hold. Assume that

$$
\left|\begin{array}{ccccc}
1 & \frac{b_{2}}{2 b_{1}} \rho_{12} & \frac{b_{3}}{2 b_{1}} \rho_{13} & \ldots & \frac{b_{n}}{2 b_{1}} \rho_{1 n} \\
\frac{b_{1}}{2 b_{2}} \rho_{21} & 1 & \frac{b_{3}}{2 b_{2}} \rho_{23} & \ldots & \frac{b_{n}}{2 b_{2}} \rho_{2 n} \\
\vdots & \vdots & \vdots & \ddots & \vdots \\
\frac{b_{1}}{2 b_{n}} \rho_{n 1} & \frac{b_{2}}{2 b_{n}} \rho_{n 2} & \frac{b_{3}}{2 b_{n}} \rho_{n 3} & \ldots & 1
\end{array}\right| \neq 0 .
$$

Let the risk model be described by equation (11). If the investment strategy $\widehat{K}^{i}$ is constant, that is, if

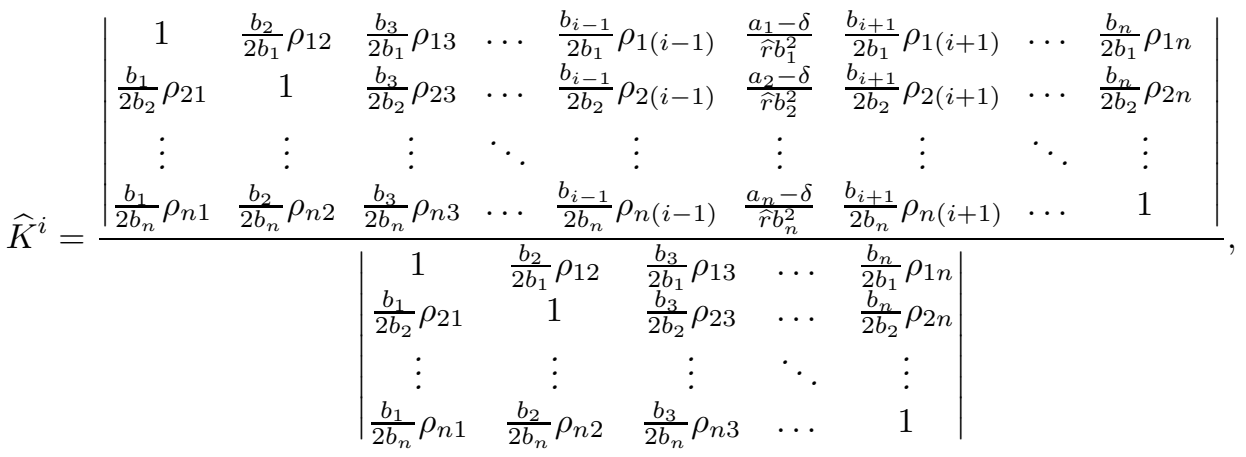

$i=1, \ldots, n$, then the following upper bound holds for the ruin probability:

$$
\mathrm{P}\{\tau(u)<\infty\} \leq e^{-\widehat{r} u},
$$

where $\widehat{r}$ is a unique positive root of the equation

$$
\beta h(\widehat{r})=\sum_{i=1}^{n} \frac{\widehat{r}^{2} b_{i}^{2}\left(\widehat{K}^{i}\right)^{2}}{2}+\widehat{r} \cdot \inf _{x \geq 0}\{p(x)+\delta x\} .
$$


Proof. If the investment strategy (23) is constant, then conditions (K1) and (K2) are obviously satisfied. Condition (K3) also holds in this case, since (K3) follows from the inequality

$$
\beta h(r) \leq \sum_{i=1}^{n} \frac{r^{2} b_{i}^{2}\left(\widehat{K}^{i}\right)^{2}}{2}+r \inf _{x \geq 0}\{p(x)+\delta x\}
$$

according to (20).

The maximal value of $r$ for which inequality (26) holds is the one that turns (26) to an equality. This value coincides with a solution of equation (25).

The left hand side of (25) exponentially increases and is a concave function of $r$ that passes the origin, while the right hand side of (25) is a linear function of $r$ that crosses the $x$-axis at some nonnegative point. Thus there exists a unique intersection point of the graphs of these functions and the abscissa of this point is positive. To prove the linearity of the right hand side of (25) one can substitute the explicit expression from (23) for the strategy and expand the determinant in (23) with respect to the column $i$.

Assumptions (R1)-(R3) of Theorem 1 follow from the Gronwall inequality applied to an arbitrary interval $[0, T]$ (see [11, p. 192]).

Let us, for example, prove the inequality

(R1) $\mathrm{P}\left\{\int_{0+}^{t \wedge \tau(u)} e^{-r R_{s-}}\left|R_{s}\right| d s<\infty, i=1, \ldots, n\right\}=1, t \in[0, T]$.

We find the upper bound for the process $\left|R_{s}\right|, s \in[0, t \wedge \tau(u) \wedge T]$, by using representation (3), the Gronwall inequality, and property (P1). According to (P1), the function $p(\cdot)$ is bounded; that is, there exists a constant $C>0$ such that $p(x) \leq C$ for all $x \in[0, t \wedge \tau(u) \wedge T]$ and

$$
\begin{aligned}
\left|R_{s}\right| & =R_{s} \leq u+C s+\sum_{i=1}^{n}\left(a_{i}-\delta\right) s \widehat{K}^{i}+\delta \int_{0}^{s} R_{z} d z+\sum_{i=1}^{n} b_{i} s \widehat{K}^{i}\left|W_{s}^{i}-W_{0}^{i}\right| \\
& \leq H(\omega) \cdot e^{\delta s}, \quad s \in[0, T],
\end{aligned}
$$

where

$$
H(\omega):=u+\left(C+\sum_{i=1}^{n}\left(a_{i}-\delta\right) \widehat{K}^{i}\right) s+\sum_{i=1}^{n} b_{i} \widehat{K}^{i} \cdot \max _{s \in[0, T]}\left|W_{s}^{i}\right| .
$$

Therefore

$$
\int_{0+}^{t \wedge \tau(u) \wedge T} e^{-r R_{s-}}\left|R_{s}\right| d s \leq \int_{0+}^{t \wedge \tau(u) \wedge T} H(\omega) e^{\delta s} d s<\infty \quad \text { almost surely; }
$$

that is, condition $(\mathrm{R} 1)$ holds on the interval $[0, \tau(u) \wedge T]$.

Conditions (R2) and (R3) can be proved in a similar way for the interval $[0, \tau(u) \wedge T]$.

Since $T>0$ is an arbitrary constant, all the assumptions of Theorem 1 hold for the interval $[0, \tau(u)]$. According to Theorem 1 and Remark 2 the process $\left\{X_{t \wedge \tau(u)}\right\}$ is a supermartingale with respect to the flow of $\sigma$-algebras $\mathcal{F}_{t}$.

Now we use a standard argument to get an upper bound for the ruin probability of an insurance company:

$$
\begin{aligned}
e^{-\widehat{r} u} & =X_{0}(u, \widehat{K}, \widehat{r}) \geq X_{t \wedge \tau(u)}(u, \widehat{K}, \widehat{r})=X_{\tau(u)}(u, \widehat{K}, \widehat{r}) \cdot I_{\tau(u)<t}+X_{t}(u, \widehat{K}, \widehat{r}) \cdot I_{\tau(u) \geq t} \\
& \geq X_{\tau(u)}(u, \widehat{K}, \widehat{r}) \cdot I_{\tau(u)<t}
\end{aligned}
$$

and

$$
\lim _{t \rightarrow \infty} \mathrm{E} X_{\tau(u)}(u, \widehat{K}, \widehat{r}) \cdot I_{\tau(u)<t}=\mathrm{E} X_{\tau(u)}(u, \widehat{K}, \widehat{r}) \cdot I_{\tau(u)<\infty}
$$


Thus

$$
\begin{aligned}
e^{-\widehat{r} u} & \geq \mathrm{E}\left(X_{\tau(u)}(u, \widehat{K}, \widehat{r}) / \tau(u)<\infty\right) \cdot \mathrm{P}\{\tau(u)<\infty\} \\
& \Rightarrow \mathrm{P}\{\tau(u)<\infty\} \leq \frac{e^{-\widehat{r} u}}{\mathrm{E} X_{\tau(u)}(u, \widehat{K}, \widehat{r})} \leq e^{-\widehat{r} u} .
\end{aligned}
$$

The latter inequality follows from $R_{\tau(u)}<0$. Theorem 2 is proved.

Remark 3. If $p(x):=p$ and $p=$ const, then the upper bound for the ruin probability is given by

$$
\mathrm{P}\{\tau(u)<\infty\} \leq e^{-\widehat{r} u}
$$

where $\widehat{r}$ is a unique positive root of the equation

$$
\beta h(\widehat{r})=\sum_{i=1}^{n} \frac{\widehat{r}^{2} b_{i}^{2}\left(\widehat{K}^{i}\right)^{2}}{2}+\widehat{r} p .
$$

Since

$$
\sum_{i=1}^{n} \frac{\widehat{r}^{2} b_{i}^{2}\left(\widehat{K}^{i}\right)^{2}}{2}>0
$$

the latter bound improves the classical estimate

$$
\mathrm{P}\{\tau(u)<\infty\} \leq e^{-\widetilde{r} u}
$$

where $\widetilde{r}$ is a unique positive root of the equation

$$
\beta h(\widetilde{r})=\widetilde{r} p .
$$

Remark 4. The case of $n=1$ covers the results of [7]. Indeed, if condition (P1) holds, the risk model is described by equation (11), and if the investment strategy $\widehat{K}$ is constant,

$$
\widehat{K}=\frac{a-\delta}{\widehat{r} b^{2}},
$$

then the ruin probability is estimated by

$$
\mathrm{P}\{\tau(u)<\infty\} \leq e^{-\widehat{r} u},
$$

where $\widehat{r}$ is a unique positive root of the equation

$$
\beta h(\widehat{r})=\frac{(\delta-a)^{2}}{2 b^{2}}+\widehat{r} \cdot \inf _{x \geq 0}\{p(x)+\delta x\} .
$$

Example 1. Let $n=2$ and $\rho_{12} \neq \pm 2$. Assume that an insurance company follows the following constant investment strategy:

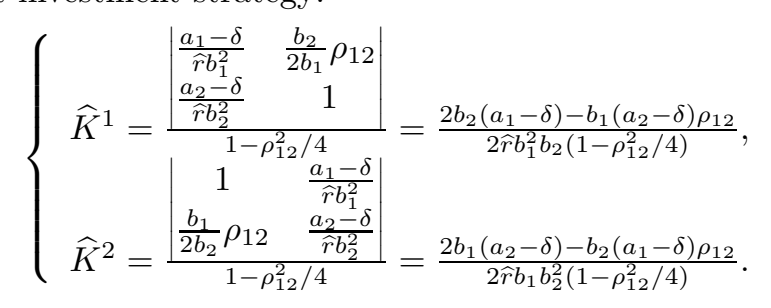

Then the following upper bound holds for the ruin probability:

$$
\mathrm{P}\{\tau(u)<\infty\} \leq e^{-\widehat{r} u}
$$

where $\widehat{r}$ is a positive root of the equation

$$
\begin{aligned}
\beta h(\widehat{r})= & \frac{\left(\left(2 b_{2}\left(a_{1}-\delta\right)-\rho_{12} b_{1}\left(a_{2}-\delta\right)\right)^{2}+\left(2 b_{1}\left(a_{2}-\delta\right)-\rho_{12} b_{2}\left(a_{1}-\delta\right)\right)^{2}\right)}{2\left(4-\rho_{12}^{2}\right) b_{1}^{2} b_{2}^{2}} \\
& +\widehat{r} \cdot \inf _{x \geq 0}\{p(x)+\delta x\} .
\end{aligned}
$$


It is clear that the above equation has a positive root, since the function on the left hand side is convex and passes the origin, while the function on the right hand side is linear with respect to $r$ and crosses the $x$-axis at a positive point.

Remark 5. Similar to the paper [7] and using the results of [12], one can prove that if $p(\cdot) \in \mathcal{C}(\mathbb{R})$ or if $p(\cdot) \in \mathcal{B} \mathcal{V}_{\text {loc }}\left(\mathbb{R}_{+}\right)$, then equation (1) has a solution for an arbitrary collection of strategies $\left\{K^{i}, i=1, \ldots, n\right\}$ and for an arbitrary interval $[0, T]$, provided that $\rho_{i j}=0, i=1, \ldots, n, j=1, \ldots, n$.

\section{CONCLUding REMARKS}

Sufficient conditions are found in the paper under which the exponent of a risk process is a local martingale and an upper bound is obtained for the ruin probability of an insurance company. The company is allowed to use its capital and buy a finite number of risky assets and to change the insurance premiums. The upper bound obtained in this paper improves the Cramér-Lundberg estimate for the classical risk model.

\section{BIBLIOGRAPHY}

1. J. Gaier, P. Grandits, and W. Schachermayer, Asymptotic ruin probabilities and optimal investment, Ann. Appl. Probab. 13 (2003), 1054-1076. MR1994044 (2004k:91124)

2. Q. Tang, Asymptotic Ruin Probabilities of the Renewal Model with Constant Interest Force and Regular Variation, Technical Report, No. 9/04, 2004. MR2118521 (2005k:62241)

3. J. Cai and D. C. M. Dickson, Upper Bounds for Ultimate Ruin Probabilities in the Sparre Andersen Model with Interest, Research paper number 97, Melbourne, Insurance Math. Econom. 32 (2003), 61-71. 2002. MR.1958769 (2003k:60046)

4. J. Grandell, Aspects of Risk Theory, Springer-Verlag, New York, 1991. MR.1084370|(92a:62151)

5. C. Kluppelberg, A. E. Kyprianou, and R. A. Maller, Ruin probabilities and overshoots for central Lévy insurance risk processes, Ann. Appl. Probab. 14 (2004), 1766-1801. MR2099651 $(2005 \mathrm{j}: 60097)$

6. J. Paulsen, J. Kasozi, and A. Steigen, A numerical method to find the probability of ultimate ruin in the classical risk model with stochastic return on investments, Insurance Math. Econom. 36 (2005), no. 3, 399-420. MR2152852 (2006b:60148)

7. M. O. Androshchuk and Yu. S. Mishura, An estimate of the ruin probability for an insurance company investing in a BS-market, Ukrain. Matem. Zh. 59 (2007), no. 11, 1143-1153. (to appear) MR 2402185

8. S. Asmussen, Ruin Probabilities, World Scientific Publishing Co., Inc., River Edge, NJ, 2000. MR $1794582(2001 \mathrm{~m}: 62119)$

9. P. E. Protter, Stochastic Integration and Differential Equations, Springer, Berlin, 2004. MR 2020294 (2005k:60008)

10. D. K. Faddeev and I. S. Sominskiū, Problems in Higher Algebra, "Nauka", Moscow, 1972; English transl., W. H. Freeman and Co., San Francisco-London, 1965. MR0176990 (31:1258)

11. R. J. Elliott, Stochastic Calculus and Applications, Springer, New York, 1982. MR678919 (85b:60059)

12. T. A. Kiffe, A Discontinuous Volterra Integral Equation, J. Integral Equations 1 (1979), 193200. MR.540826 (80i:45006)

Department of Probability Theory and Mathematical Statistics, Faculty for Mechanics and Mathematics, National Taras Shevchenko University, Academician Glushkov Avenue 6, KYIV 03127, UKRAINE

E-mail address: andr_m@univ.kiev.ua

Received 28/JUL/2006

Translated by O. I. KLESOV 